\title{
ASPECTOS DA GRAVIDEZ E PÓS-PARTO DE ADOLESCENTES PORTADORAS DE FEBRE REUMÁTICA
}

\author{
Ana Julia Pantoja Moraes, Pollyana Maria ferreira Soares, Marta Miranda leal, Adriana Maluf Elias Sallum, \\ Ana Paola Navarrete Lotito, Clovis Artur Almeida Silva* \\ Trabalho realizado nas unidades de Reumatologia Pediátrica e Adolescência do Instituto da Criança, HC-FMUSP, São Paulo, SP.
}

RESUMO - OBjetivo. Avaliar a incidência e evolução de gravidez entre adolescentes portadoras de febre reumática (FR) do nosso serviço.

Métodos. Avaliação retrospectiva de 510 prontuários de pacientes com diagnóstico de FR, no período de 1983 a 2001.

Resultados. Dos 510 pacientes analisados, 123 (46\%) eram adolescentes femininas. Dezesseis (13\%) engravidaram neste período, com um total de 19 gestações (uma apresentou duas gestações e outra três); 14 realizaram pré-natal adequadamente. $A$ idade da primeira gestação variou de 14 a 19 anos (média 16,7); e a idade do início da atividade sexual, de 13 a 18 anos (média 15,2 ). Insuficiência mitral ocorreu em I5 casos, estando associada com insuficiência aórtica em cinco. Intercorrências durante o pré-natal foram observadas em duas pacientes: em uma houve recidiva da FR com Coréia e em outra infecção pelo HIV. 0 parto vaginal ocorreu em sete adolescentes, parto fórcipe em três e cesareana em quatro: uma com HIV, uma com gestação gemelar e duas com distocia funcional. Treze recém-nascidos foram adequados para idade gestacional e apenas os gêmeos foram prematuros. No pós-parto, uma paciente apresentou infecção na incisão cirúrgica e outra abscesso mamário. Nenhuma paciente reativou a FR no parto e pós-parto.

CONCLUSÕEs. As gestações não apresentaram descompensação cardíaca, com predomínio de valvulites de leve intensidade. Atividade sexual precoce e aumento da gravidez em adolescentes são realidades nos ambulatórios de reumatologia pediátrica, justificando a necessidade de uma melhor orientação em relação à sexualidade e uso de métodos anticoncepcionais na rotina do atendimento.

Unitermos: Febre reumática. Gravidez. Adolescência. Assistência pré-natal.

\section{INTRODUÇÃo}

A febre reumática (FR) assume proporções alarmantes, não só em razão da sua freqüência, mas principalmente pela gravidade do comprometimento cardíaco e conseqüentemente a um grau de incapacidade física e mortalidade', o que a torna um dos principais problemas de saúde pública nacional, com um alto ônus financeiro ao serviço de saúde brasileiro ${ }^{2}$. Atualmente, pelo aumento da sobrevida desses pacientes, os ambulatórios de reumatologia pediátrica têm tido que lidar com uma população cada vez maior de adolescentes e, portanto, com questões próprias da adolescência, entre elas: o desenvolvimento da sexualidade, o início cada vez mais precoce da atividade sexual e suas possíveis conseqüências como doenças sexualmente transmissíveis (DST) e gravidez.

\footnotetext{
*Correspondência:
}

Unidade de Reumatologia Pediátrica do ICr-HC-FMUSP

Rua Dr. Enéas Carvalho de Aguiar, 647

CEP - 05403-900 - São Paulo - SP - Brasil

Tel.: (11) 3069-8675/8510

clovisaas@icr.hcnet.usp.br
Um estudo realizado na população brasileira demonstrou que 42,5\% das mulheres jovens iniciam sua atividade sexual antes dos 18 anos de idade e, em 1996, cerca de um quarto dos partos realizados no país foram em adolescentes ${ }^{3}$. As características da adolescência com crescimento e desenvolvimento físico incompletos, mudanças no processo de socialização e a organização da personalidade configuram à gestação adolescente uma complexidade muito grande, acarretando agravos físicos e psicossociais para mães e filhos ${ }^{4}$. Além disso, observa-se retardo do início do pré-natal e menor freqüência ao acompanhamento da gravidez entre as adolescentes, provavelmente pela falta de maturidade necessária nesta situação. $\mathrm{Na}$ gestação de uma jovem com cardiopatia reumática, esses fatores agravantes adquirem dimensão ainda maior, uma vez que pode haver piora da cardiopatia, com conseqüências maternas, fetais e no recém-nascido ${ }^{5,6}$.

Este trabalho tem por objetivo avaliar a incidência e evolução da gravidez em adolescentes portadoras de febre reumática, acompanhadas no nosso ambulatório de reumatologia pediátrica, e verificar a evolução e repercussões dessas gestações.

\section{Métodos}

Foi realizada uma avaliação retrospectiva de prontuários de 510 pacientes com febre reumática diagnosticados de acordo com os critérios de Jones modificados (1992)7, no período de 1983 a 200 I (pacientes diagnosticados antes de 1992 foram reavaliados quanto aos critérios de Jones (1992). Objetivou-se identificar as adolescentes que engravidaram durante este período para preenchimento de um protocolo a partir dos dados destes prontuários e de informações obtidas por telefone destas jovens.

Dados epidemiológicos (idade na primeira, segunda e terceira gestação, tempo de doença à gestação, parto e atividade de doença durante a gestação), características clínicas dos surtos (inicial e recorrências) e aderência à profilaxia secundária (a cada 21 dias) foram analisados.

Pacientes não aderentes foram definidas neste estudo como: aquelas que apresentaram recorrências da doença (alterações clínicas/ laboratoriais compatíveis com FR e/ou novas lesões valvulares cardíacas ou piora destas no ecocardiograma) ou uso irregular de penicilina (informação e registro no cartão). 
Os dados do ECO/Doppler, realizado em todas pacientes, determinaram o envolvimento valvar ${ }^{8}$. Os títulos de anti-estrep tolisina $\mathrm{O}$ (ASLO), dosados na maioria dos pacientes, foram considerados alterados quando houve um aumento de duas vezes ou mais em relação ao título inicial ou quando o título inicial foi igual ou superior a $750 \mathrm{UI} / \mathrm{ml}$ (em caso de dosagem única) 9 .

A história gestacional foi avaliada de acordo com a idade na primeira gestação, atividade da doença neste período e características do parto, pós-parto e do recém-nascido.

Obteve-se, pelo telefone, informação sobre a idade de início da atividade sexual, número e idade dos parceiros.

O projeto foi aceito pela Comissão de Pesquisa e Ética do HC-FMUSP (CAPPesq $95 \mathrm{I} / 0 \mathrm{I}$ ). O termo de consentimento livre e esclarecido foi assinado pelas pacientes grávidas no momento do estudo.

\section{Resultados}

Dos 510 prontuários estudados, 267 (52\%) eram do sexo feminino e 243 (48\%) do sexo masculino (relação masculino:feminino I:I,09). Entre as 267 pacientes, 123 (46\%) tinham entre 10 a 19 anos. Dezesseis adolescentes (13\%) engravidaram com um total de dezenove gestações, pois uma paciente apresentou duas (paciente 7) e outra três gravidezes (paciente 2), ainda na adolescência (Tabela I). Quatorze jovens realizaram acompanhamento pré-natal adequado e duas abandonaram o seguimento durante a gestação (pacientes 15 e 16). Com estas duas pacientes não foi possível obter informações sobre o parto.

As características clínicas, critérios de febre reumática na época do diagnóstico, aderência e recorrência antes da gravidez estão apresentadas na Tabela 2. A idade de início da doença, nas 16 pacientes estudadas, variou de 6 a 12 anos (média 9,5 anos). Cardite foi o sinal mais freqüentemente observado, a coréia isolada foi evidenciada como critério maior de Jones ao diagnóstico em uma paciente e nas demais observaram-se combinações variadas de cardite, artrite e coréia. As lesões valvares encontradas foram: insuficiência mitral em oito pacientes, insuficiência mitral e aórtica em cinco e regurgitação mitral com espessamento de valva mitral em duas. A função miocárdica (fração de ejeção) foi normal em todas os pacientes com lesões valvares. A elevação de

Tabela I - Idade na primeira, segunda e terceira gestação, tempo de doença à gestação, parto e atividade de doença durante a gestação em 16 pacientes com febre reumática

\begin{tabular}{|c|c|c|c|c|}
\hline Paciente & $\begin{array}{c}\text { Idade na gestação } \\
\text { Anos } \\
I^{\mathrm{a}}, 2^{\mathrm{a}}, 3^{\mathrm{a}}\end{array}$ & $\begin{array}{c}\text { Tempo de doença } \\
\text { à gestaçãa Anos } \\
1^{\mathrm{a}}, 2^{\mathrm{a}}, 3^{\mathrm{a}}\end{array}$ & $\begin{array}{l}\text { Parto } \\
1^{\circ}, 2^{\circ}, 3^{\circ}\end{array}$ & $\begin{array}{c}\text { Atividade da } \\
\text { doença durante } \\
1^{\mathrm{a}}, 2^{\mathrm{a}}, 3^{\mathrm{a}}\end{array}$ \\
\hline I & 16 & 9 & Vag & - \\
\hline 2 & $14,15,19$ & $3,4,8$ & Vag, Vag, Vag & t \\
\hline 3 & 18 & 6 & Cesáreo & - \\
\hline 4 & 17 & 9 & Fórcipe & - \\
\hline 5 & 17 & 7 & Vag & - \\
\hline 6 & 14 & 3 & Fórcipe & - \\
\hline 7 & 16,17 & 7,8 & Vag, Vag & - \\
\hline 8 & 19 & 13 & Fórcipe & - \\
\hline 9 & 15 & 4 & Cesáreo & - \\
\hline 10 & 19 & 11 & Cesáreo & - \\
\hline 11 & 16 & 7 & Vag & - \\
\hline 12 & 16 & 5 & Vag & - \\
\hline 13 & 19 & 7 & Cesáreo & - \\
\hline 14 & 15 & 5 & Vag & - \\
\hline 15 & 17 & 6 & Sl & Sl \\
\hline 16 & 18 & 12 & SI & Sl \\
\hline
\end{tabular}

Tabela 2 - Características clínicas, critérios de febre reumática na época do diagnóstico, aderência e recorrência antes da gestação $(n=16)$

\begin{tabular}{|c|c|c|c|c|c|c|}
\hline \multirow[t]{2}{*}{ Paciente } & \multirow{2}{*}{$\begin{array}{l}\text { Idade } \\
\text { Anos }\end{array}$} & \multicolumn{2}{|c|}{ Critérios de Jones } & \multirow[t]{2}{*}{ ASLO } & \multirow[t]{2}{*}{ Aderência } & \multirow[t]{2}{*}{ Recorrência } \\
\hline & & Maior & Menor & & & \\
\hline | & 7 & $A r, I M L$ & Febre, VHS & - & - & - \\
\hline 2 & II & $\mathrm{Ar}, \mathrm{Co}, \mathrm{IML}, \mathrm{IAOL}$ & Febre,VHS & + & - & + \\
\hline 3 & 12 & $\mathrm{Ar}, \mathrm{Co}, \mathrm{IML}, \mathrm{IAOM}$ & VHS & - & - & + \\
\hline 4 & 8 & Co, IML & 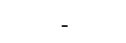 & - & + & - \\
\hline 5 & 10 & $\mathrm{Ar}, \mathrm{Co}, \mathrm{IML}$ & VHS & + & t & - \\
\hline 6 & II & $\mathrm{Ar}, \mathrm{Co}, \mathrm{IML}$ & VHS & + & - & t \\
\hline 7 & 9 & Co & - & + & + & - \\
\hline 8 & 6 & 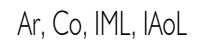 & VHS, PCR & + & - & + \\
\hline 9 & II & Co, IML & - & + & + & - \\
\hline 10 & 8 & $\mathrm{Ar}, \mathrm{Co}, \mathrm{IML}, \mathrm{IAoL}$ & VHS, PCR & + & - & t \\
\hline II & 9 & Ar, IML, IAoL & VHS, PCR & + & + & - \\
\hline 12 & II & $\mathrm{Co}, \mathrm{IML}$ & VHS & - & - & + \\
\hline 13 & 12 & Co, IML & - & + & - & + \\
\hline 14 & 10 & Co, IML & - & - & t & - \\
\hline 15 & II & Co, IML & - & - & + & - \\
\hline 16 & 6 & Co, Ar, IML & - & - & t & - \\
\hline
\end{tabular}

$\mathrm{Ar}=$ artrite, $\mathrm{ASLO}=$ antiestreptolisina $\mathrm{O}, \mathrm{CO}=$ Coréia, $\mathrm{PCR}=$ proteína $\mathrm{C}$ reativa, $\mathrm{VHS}=$ velocidade de hemossedimentação, $L=$ leve, $M=$ moderada, $I M=$ insuficiência mitral, $\mid A O=$ insuficiência aórtica

proteína $\mathrm{C}$ reativa (PCR) e/ou velocidade de hemossedimentação (VHS) foram evidenciadas em nove pacientes (56\%). ASLO foi positiva em nove pacientes (56\%). Oito pacientes não aderiram à profilaxia secundária $(50 \%)$ e, destas, sete $(44 \%)$ apresentaram recidivas com coréia e cardite anteriores à gestação.

As características na primeira gravidez são apresentadas na Tabela I. A idade da primeira gestação variou de 14 aos 19 anos (média 16,7 anos). O tempo de seguimento da doença até a primeira gestação variou de 3 a 13 anos (média de sete anos). A idade do início da atividade sexual variou de 13 a 18 anos (média de 15,2 anos). As intercorrências durante o pré-natal foram observadas em duas pacientes: 
uma apresentou recidiva da FR com coréia (paciente 2), e em outra foi diagnosticada infecção pelo HIV (paciente 10). O parto vaginal ocorreu em sete adolescentes (50\%); parto fórcipe em três (21,5\%); e parto cesáreo em quatro pacientes (28,5\%): uma com HIV, outra com gestação gemelar com trabalho de parto prematuro e cardiopatia reumática moderada (30 semanas de idade gestacional) e duas com distocia funcional por trabalho de parto prolongado. Treze recém-nascidos foram adequados para idade gestacional e os gêmeos foram prematuros. No pós-parto, uma paciente apresentou infecção na incisão cirúrgica (paciente 3) e outra abscesso mamário (paciente 8), sendo necessário uso de antibiótico endovenoso em ambas. Nenhuma paciente evoluiu com descompensação cardíaca ou reativou a FR no parto e pós-parto.

A idade dos parceiros variou de 16 a 24 anos (média 19 anos). A maioria referiu um único parceiro, exceção feita às jovens que apresentaram mais de uma gestação (pacientes 2 e 7 ), onde cada gravidez foi fruto de um relacionamento diferente.

\section{Discussão}

As pacientes com FR tornam-se adolescentes e adultos jovens com vida sexual ativa e têm a possibilidade de constituir família. A escassez de publicações, em literatura médica, sobre a sexualidade e função gonadal das adolescentes com FR estimulou a realização deste estudo.

A idade de início da atividade sexual variou de 13 a I 8 anos (média I5,2 anos), compatível com os dados da população brasileira, cuja idade mediana à primeira relação sexual é de 15 anos $^{3}$. A redução da idade de início da vida sexual encontra-se entre os principais fatores responsáveis pelo aumento da gravidez na adolescência, juntamente com menarca mais precoce, influência da mídia e a fragilidade de um projeto pessoal de vida ${ }^{10}$.

A gravidez na adolescência é um problema universal atingindo tanto países desenvolvidos quanto subdesenvolvidos, embora principalmente estes últimos. No Brasil, em 1997, mais de 700.000 partos (26\% do total) foram em mulheres com menos de 20 anos de idade $"$. Mesmo representando um número pequeno frente ao universo de adolescentes brasileiras que já engravidaram, o encontro neste trabaTho de 16 adolescentes grávidas, ou seja, 13\% da clientela adolescente feminina portadora de $F R$, é preocupante e expressa os riscos a que estão sujeitas.

Na nossa Unidade de Reumatologia Pediátrica tem sido observado um aumento da freqüência de gravidez em adolescentes portadoras de doenças crônicas reumatológicas, refletindo o que está acontecendo na sociedade como um todo. Trabalho realizado com 43 adolescentes com lúpus eritematoso sistêmico juvenil, seguidas no nosso serviço durante os anos de 1999 e 2000, demonstrou a ocorrência de gravidez em seis $(14 \%)^{12}$.

Nos EUA, anualmente, $8 \%$ das adolescentes sexualmente ativas engravidam com 14 anos de idade, $18 \%$ entre $15-17$ anos e $22 \%$ entre $18-19$ anos $^{13}$. No Brasil, $18 \%$ de toda a população feminina de 15 a 19 anos já engravidou alguma vez ${ }^{3}$. No presente estudo, a idade média da primeira gravidez foi de 16,3 anos, e a idade média dos parceiros foi de 19 anos, ressaltando a precocidade do evento.

É interessante comentar a reincidência de gravidez em dois casos e a ocorrência de DST em outro. Os profissionais que lidam com adolescentes sabem que a experiência de uma gravidez não planejada não é fator de proteção para prevenir que outras gravidezes aconteçam, uma vez que os fatores determinantes $d a$ primeira geralmente mantêm-se presentes, não sendo raro a ocorrência de outras gestações, também não planejadas, ainda na adolescência. Esse fenômeno é observado nesta casuística (pacientes 2 e 7). A prevenção de uma outra gestação adolescente deve ser uma das prioridades no atendimento dessas jovens mães. Outra prioridade no atendimento dos adolescentes é a prevenção de DST/HIV: sabe-se que um quarto das DST acontece na adolescência e a mais alta taxa de novas infecções pelo HIV ocorre entre os jovens ${ }^{14}$.

Nesta casuística, o ciclo gravídico-puerperal evoluiu sem descompensação cardíaca, uma vez que as pacientes eram oriundas de um Serviço de Reumatologia Pediátrica que acompanha pacientes com $F R$ com várias manifestações associadas (artrite, cardite e/ou coréia) e que encaminha para seguimento no ambulatório de cardiologia aqueles com grave comprometimento cardíaco. Eis porque as adolescentes deste estudo apresentavam lesões valvares leves, com função miocárdica normal, justificando, provavelmente, a evolução favorável da gravidez. No presente estudo, $40 \%$ destas pacientes apresentaram recorrências da FR anteriormente à gravidez, conseqüentes a não aderência à profilaxia secundária com penicilina benzatina; resultados semelhantes foram relatados por Gordis et al ${ }^{15}$ que evidenciaram ausência de aderência em até 36\% dos casos com FR, acompanhados ambulatorialmente. Scheibel et al. ${ }^{16}$ encontraram maior adesão ao tratamento da FR em pacientes menores de 16 anos e atribuíram esse achado à presença dos pais ou responsáveis às consultas. Apenas uma paciente apresentou reativação da FR com coréia, pelo abandono da profilaxia secundária com penicilina benzatina, acreditando que a mesma não pudesse ser utilizada durante a gravidez.

O fato das pacientes serem portadoras de patologias cardíacas leves, provavelmente justifica a maior freqüência de parto vaginal e a necessidade de fórceps de alívio em cerca de um quinto dos casos. No estudo de Ávila e Grinberg 5 , com l.000 grávidas portadoras de cardiopatias das mais comuns, como prolapso de valva mitral, até as mais graves como miocardiopatia chagásica, o tipo de parto mais freqüente também foi o vaginal (53\%), sendo 27,3\% com o auxílio de fórceps de alívio; o parto cesárea foi realizado por razões estritamente obstétricas ou por situações cardíacas especiais que recomendavam sua antecipação. Gómez et al. ${ }^{17}$ estudaram 80 pacientes grávidas, com idade variando de 18 a 42 anos, portadoras de cardiopatia reumática; insuficiência mitral foi encontrada em $80 \%$ e o tipo de parto mais freqüente foi o vaginal em $87,5 \%$ das gestantes, semelhantes aos resultados do presente estudo.

A ocorrência de complicações infecciosas no período puerperal em 14,3\% dos casos (duas pacientes) é superior aos dados do DATASUS ${ }^{18}$, onde a incidência de complicações relacionadas com o puerpério é em torno de $0,6 \%$ para o período de 1995 a 2001 . O pequeno número de casos provavelmente justifica a alta incidência encontrada neste estudo.

Os dados apresentados chamam a atenção para a precocidade da atividade sexual e incidência da gravidez em adolescentes portadoras de FR. Quando se compreende que o desenvolvimento da sexualidade envolve questões que passam pelo papel da socialização, da maturidade física, da imagem corporal, da auto-estima, das aspirações referentes ao futuro e da construção da própria identidade, 
consegue-se entender como a doença interfere nesse processo e é um determinante de risco ${ }^{10}$. As dificuldades de ser aceita pelo grupo, a família superprotetora, as internações, as restrições e rotinas impostas pela doença e seu tratamento dificultam o processo adolescente. Acresce-se ainda o fato de que, entre as jovens com doenças crônicas, o desejo de gravidez, mesmo que inconsciente, pode surgir como forma de provar a capacidade de gerar vida, carregando-a dentro de si, ou como tentativa de resgate da própria vida ${ }^{19}$.

O médico especialista tem um papel fundamental, pois, muitas vezes, é a única referência de profissional de saúde na orientação sexual destes pacientes. Dessa forma 0 reumatologista pediátrico precisa criar espaços dentro da sua consulta onde ele tenha a possibilidade de abordar e discutir aspectos da sexualidade, orientar métodos contraceptivos e alertar quanto aos riscos e à prevenção de doenças sexualmente transmissíveis.

\section{Conclusóes}

Atividade sexual precoce (idade média de 15 anos) e o encontro de $18 \%$ de gravidez em adolescentes com FR são realidades nos ambulatórios de reumatologia pediátrica, justificando a necessidade de uma melhor orientação em relação à sexualidade e uso de métodos anticoncepcionais na rotina do atendimento. As pacientes apresentaram, predominantemente, valvulites leves (com função miocárdica normal) evoluindo sem descompensação cardíaca e reativação da FR durante a gravidez.

\section{Agradecimentos}

Os autores agradecem às Dras. Maria Helena B. Kiss, Bernadete L. Liphaus e Lucia M. M. A. Campos; à equipe do Ambulatório de Gravidez de Alto Risco do HC-FMUSP pelo seguimento de cinco pacientes.

\section{Conflito de interesse: não há}

\section{SUMMARY}

\section{AspeCts OF THE PREGNANCY AND POST DELIVERY OF ADOLESCENTS WITH RHEUMATIC FEVER}

BACKGROUND. To evaluate the incidence and follow-up of pregnancy among adolescents with rheumatic fever (RF) attended at the authors' service.
Methods. A retrospective evaluation was undertaken of 510 patients with a diagnosis of RF according to records from 1983 to 2001 .

REsULTS. We evaluated 510 patients, 123 (43\%) were female adolescents. Sixteen (13\%) patients became pregnant during this period, with a total of 19 gestations (one presented two gestations and another three); 14 realized the prenatal care appropriately. Age of the first gestation ranged from 14 to 19 years (mean 16.7); and age at the beginning of sexual activity ranged from 13 to 18 years (mean | 5.2). Mitral insufficiency occurred in 15 cases associated with aortic insufficiency in 5 . Intercurrent disease during prenatal care was observed in two patients: in one there was recurrence of RF with chorea and in the other HIV infection. Vaginal delivery occurred in seven adolescents, forceps delivery in three and cesarean in four: one with $H I V$, one with twin pregnancy and two with functional dystocia. Thirteen newborn were adequate for gestational age and only the twins were premature. In the postpartum, one patient presented infection in the surgical incision and another had mammary abscess. No patient reactivated RF in childbirth or postpartum.

Conclusions. Pregnancies did not present cardiac decompensation, there was however predominance of mild valvulitis. Precocious sexual activity and greater incidence of pregnancy among adolescents are realities in the pediatric rheumatology clinics; consequently there is a need for improved orientation in relation to sexuality and use of birth-control methods in the routine of such services. [Rev Assoc Med Bras 2004; 50(3): 293-6]

KeY WoRDs: Rheumatic fever. Pregnancy. Adolescence. Pre-natal care.

\section{REFERÊNCIAS}

I. Sbaffi F. Papel do pediatra na prevenção da febre reumática. Arq Bras Pediat 1995; 2:123-5.

2. Terreri MT, Ferraz MB, Goldenberg J, Len CA, Hilário MOE. Resource utilization and cost of rheumatic fever. J Rheumatol 200 I; 28: 1394-7.

3. Badiani R, Quental I, Santos EM. DST/AIDS e a pesquisa nacional sobre demografia e saúde: uma análise do nível de conhecimento e comportamento de vulnerabilização. Rio de Janeiro: BEMFAM/DHS; 1997.

4. Sharma AK, Verma k, Khatri S, Kannan AT. Pregnancy in adolescents: Study of Risks and Outcome in Eastern Nepal. Indian Pediatr 200 । ; 38: | 405-9.
5. Ávila WS, Grinberg M. Gestação em portadoras de afecções cardiovasculares. Experiência com 1000 casos. Arq Bras Cardiol 1993; 60:5-1।.

6. Gopeesingh TD, Rahaman MB. Pregnancy complicated by maternal cardiac disease. West Indian Med J 1998; 37:218-21.

7. Dajani AS. Special writing group of the committee on rheumatic fever, endocarditis and Kawasaki disease of the council on cardiovascular disease in the young of the American Heart Association. Guidelines for the diagnosis of rheumatic fever - Jones criteria, 1992, update. JAMA 1992; 268:2069-73.

8. Hilário MOE, Andrade JL, Gasparian $A B$, Carvalho AC, Andrade CT, Len CA. The Value of Echocardiography in the diagnosis and follow-up of rheumatic carditis in children and adolescents: A 2 Year prospective study. J Rheumatol 2000; 27: 1082-6.

9. Machado CSM, Ortiz K, Martins ALB, Martins RS, Machado NC. O perfil da antiestreptolisina O (ASO) na Febre Reumática Aguda (FRA). J Pediatr (Rio de J) 200 I; 77: I 05- I I.

10. Saito MI. Sex education in school: preventing unwanted pregnancy in adolescents. Int J Gynecol Obstet 1998; 63(Suppl I): I 57-60.

I I. Serviço de Assistência à Saúde do Adolescente do Ministério da Saúde (SASAD). Prevenção intersetorial da gravidez na adolescência. Documento preliminar. São Paulo; 1997.

12. Silva CA, Leal MM, Leone C, Simone VP, Takiuti AD, Saito MI, et al. Gonadal function in adolescents and young women with juvenile systemic lupus erythematosus. Lupus 2002; 11:419-25.

13. Pinto e Silva JL, Motta ML. Gravidez na adolescência. In: Neme B, editor. Obstetrícia básica. São Paulo: Sarvier; 2000. p. I 196-20I .

14. Centers for Disease Control and Prevention. Trends in sexual risk behaviors among high school students - United States 199|-1997. MMWR Morb Motal Wkly Rep 1998; 47:36.

15. Gordis L, Markowitz M, Lilienfeld AM. Why patients don't follow medical advice: a study of children on long-term antistreptococcal prophylaxis. J Pediatr 1969; 75:957-68.

16. Scheibel et al. Avaliação da adesão 'a profilaxia secundária da febre reumática. Rev Bras Reumatol 200 I;4 I (4):220-4.

17. Gómez MG, Pérez JST, Diaz HS, Martinez EZ. Recién nacido de madre con cardiopatia. Bol Med Hosp Infant Mex 1988;45:769-75.

18. Funasa. Morbidade hospitalar do SUS por local de residência-SP. Internação segundo lista de morbidade CID 10 [online]. Disponível em: http://www.datasus.gov.Br. Acesso em: 31 mar 2004.

19. Pinto e Silva JL. Pregnancy during adolescence: wanted vs unwanted. Int J Gynecol Obstet 1998; 63(Suppl 1):151-6.

Artigo recebido: 06/06/2003

Aceito para publicação: 20/05/2004 